\title{
Aggregating set-point temperature profiles for archetype-based: simulations of the space heat demand within residential districts
}

Ina De Jaeger, Annelies Vandermeulen, Bram van der Heijde, Lieve Helsen \& Dirk Saelens

To cite this article: Ina De Jaeger, Annelies Vandermeulen, Bram van der Heijde, Lieve Helsen \& Dirk Saelens (2020): Aggregating set-point temperature profiles for archetype-based: simulations of the space heat demand within residential districts, Journal of Building Performance Simulation, DOI: $10.1080 / 19401493.2020 .1727567$

To link to this article: https://doi.org/10.1080/19401493.2020.1727567

Published online: 18 Feb 2020.

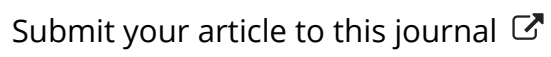

View related articles

View Crossmark data \lceil 


\title{
Aggregating set-point temperature profiles for archetype-based: simulations of the space heat demand within residential districts
}

\author{
Ina De Jaeger (iD) a,b,c , Annelies Vandermeulen (D) a,d,c, Bram van der Heijde (iD) a,d,c, Lieve Helsen (D) a, d and \\ Dirk Saelens id a,b

\begin{abstract}
${ }^{a}$ EnergyVille, Thor Park 8310, 3600 Genk, Belgium; ${ }^{b}$ KU Leuven, Department of Civil Engineering, Building Physics Section, Kasteelpark Arenberg 40,

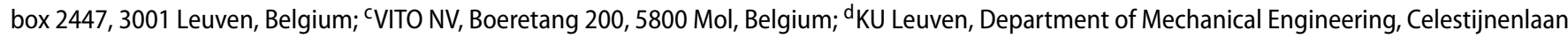
300, box 2421, 3001 Leuven, Belgium
\end{abstract}

\begin{abstract}
With the increasing integration of multiple energy carriers in district energy systems, an accurate simulation of the district energy demand becomes more crucial. To reduce the required computational power, the district energy demand is often quantified through a limited set of archetype buildings, representing the whole district. As the temporal behaviour is important to assess district energy systems, stochastic occupant models should be included in the simulation. However, obtaining representative set-point temperature profiles for archetype buildings is not straightforward, as all buildings respond differently to their demanded set-point temperature due to their thermal inertia. Hence, this paper proposes and compares three techniques to obtain the representative occupant behaviour for the archetype building, by focussing on 847 single-family dwellings. Including a smart occupant aggregation method allows to decrease the percentage error in annual energy demand for space heating between the full and the archetype simulation from around $7.5 \%$ to around $2 \%$, depending on the building model and evaluation method.
\end{abstract}

\section{ARTICLE HISTORY}

Received 11 April 2019

Accepted 4 February 2020

\section{KEYWORDS}

district energy simulations; building archetypes: stochastic occupant behaviour; space heating

\section{Introduction}

Smart district energy systems (DES) enable mutual building interactions and building grid interactions. They allow energy to be transferred from buildings with an energy excess to the grid or to buildings with an energy demand, as a result of the heterogeneity within existing districts (Lawrence et al. 2016; Kensby, Trüschel, and Dalenbäck 2015). Simulations often aid in understanding the energy flows within these smart district energy systems, providing answers and avoiding suboptimal decisions in the design and the operation of district energy systems (Allegrini et al. 2015). Within these simulations, an accurate quantification of the district energy demand in time and space is important (Mavromatidis, Orehounig, and Carmeliet 2018), requiring both building-related data and thermal and/or electrical grid characteristics.

Smart district energy systems should be assessed on different scales, each demanding a particular simulation approach. On a local scale, the location of distributed production and storage units, as well as the energy exchange between different buildings, can be studied, requiring detailed building-by-building simulations. On a larger scale, the location of central production and storage units, as well as the energy exchange between different neighbourhoods, can be studied. On this larger scale, building-by-building simulations become computationally too expensive and their level of detail is not always necessary, as was concluded by Allegrini et al. (2015).
As a result, district energy models often make use of archetypes, i.e. the building stock is categorized in a limited number of groups of buildings that are characterized by a similar energy performance and that can be represented by one archetype building (Reinhart and Davila 2016). However, the archetype buildings should always be representative for the district, as unrepresentative archetypes might result in a distorted view. For instance, De Jaeger, Reynders, and Saelens (2017) compared the use of Belgian TABULA archetypes (Cuypers et al. 2014) to the use of geospatial data for a small district of 99 buildings and concluded that the geometry of the TABULA archetypes is not representative for this particular district, resulting in significant deviations on the simulated district energy demand.

Multiple research initiatives have focused on how to define these representative archetype buildings (Swan and Ismet Ugursal 2009; Ghiassi and Mahdavi 2017; Tardioli et al. 2018). In general, archetype buildings can be either average buildings or sample buildings. In the case of average buildings, the archetype is a non-existing building, determined as the average of all cluster members. In the case of sample buildings, the archetype is an existing member of the cluster that is closest to the average building in that cluster. As archetype buildings intend to summarize the energy performance of the buildings they present, they should include the various aspects that determine the energy performance of a building: the geometry and location, the properties of the building envelope, the heating, ventilation and air

CONTACT Ina De Jaeger ina.dejaeger@kuleuven.be EnergyVille, Thor Park 8310, 3600 Genk, Belgium; KU Leuven, Department of Civil Engineering, Building Physics Section, Kasteelpark Arenberg 40, box 2447, 3001 Leuven, Belgium; VITO NV, Boeretang 200, 5800 Mol, Belgium 
conditioning (HVAC) system characteristics, possibly the renewable energy system characteristics, the building appliances and the occupant behaviour. Calculating the average value is rather straightforward for most of the building characteristics, but it is not that intuitive for the occupant behaviour, which is the focus of the present work.

Occupant behaviour is often hard to predict (Hong et al. 2017), as the occupants interact with buildings in multiple ways (Page et al. 2008). They determine the use of electrical appliances and domestic hot water, lighting utilities as well as the opening of windows. Their presence introduces both metabolic heat gains and pollutants, increasing the internal heat gains and ventilation needs respectively. Finally, occupants also set comfort requirements for the HVAC system.

Although the occupant behaviour starts to average out if the district scale increases (Baetens and Saelens 2016), it is found to introduce a significant uncertainty on the building level (Tian et al. 2018), contributing to the gap between simulated and measured energy use. To overcome this issue, various occupant behaviour models have been developed for both building and district level (Page et al. 2008; Baetens and Saelens 2016). An elaborate overview of these different occupant behaviour models is given by Happle, Fonseca, and Schlueter (2018). In general, two main occupant behaviour models can be distinguished: deterministic models and stochastic models. Deterministic models, on the one hand, include schedules and deterministic rules to specify occupant behaviour. They are often published in a standard calculation procedure for building energy demand (e.g. the ISO 13790 standard, see Roulet 2002). Stochastic models, on the other hand, typically sample from statistical distributions to predict the occurrence of particular actions within the building (e.g. Baetens and Saelens 2016). Usually, these stochastic models are based on local time-use surveys. For residential buildings, the stochastic profiles heavily depend on the number of occupants and their employment, as stated by Yao and Steemers (2005).

Deterministic occupant models are most widespread for assessing single buildings (Hong et al. 2017). They can cope with the spatial variability or diversity between buildings, but they are generally weak in capturing the temporal variability between buildings, which leads to inaccurate modelling of the district temporal behaviour (De Jaeger, Lago, and Saelens 2018a; Happle, Fonseca, and Schlueter 2018). As an example, the district peak power is usually lower than the sum of the individual building peak powers due to the temporal variability among the building occupants (Frayssinet et al. 2017), but this is generally not captured by deterministic occupant models. Happle, Fonseca, and Schlueter (2018) concluded that deterministic models perform well for monthly and yearly values due to averaging effects, but stochastic models are necessary when assessing high-resolution temporal behaviour of buildings and districts. Evins, Orehounig, and Dorer (2016) came to a similar conclusion: based on a virtual district of 500 buildings, they found that stochastic occupancy models are critical when assessing temporal effects such as peak loads and storage sizing in building-bybuilding simulations. When studying monthly or yearly values, both deterministic and stochastic models are sufficient, as long as a reliable representation of the actual occupant behaviour is provided, as shown by Tahmasebi and Mahdavi (2017). They calculated the annual heating and cooling load as well as the peak loads, based on the study of an office building, considering a different occupant for each office space within the building.

In the context of the optimal design and operation of smart district energy systems, the district temporal behaviour is of utmost importance, introducing the need for stochastic occupant models within simulations. Simultaneously, for large-scale applications, archetype-based simulations are preferred over building-by-building simulations, thanks to their lower computational load. However, obtaining an aggregated space setpoint temperature profile for use in archetype buildings is not straightforward.

As a result of the natural variability in occupant behaviour between the different buildings, their occupant behaviour profiles cannot simply be averaged. Each building reacts differently to the demanded set-point temperature, according to their thermal inertia, insulation properties, positioning of windows, etc., which should be taken into account when aggregating the occupant behaviour profiles of the individual buildings into a representative occupant behaviour profile for the archetype building. Figure 1 illustrates this conceptually. The left and middle graphs show typical temperature profiles of a poorly insulated and a well-insulated building, respectively. The right graph shows three things. It shows the average of the actual temperature profiles as a solid line at the top, the simple average of the setpoints as a dotted line and the temperature profile that is the result of the simulation of the archetype building with the average set-point as a solid line at the bottom. It is clear that in this case, the simple average will overestimate the temperature changes in the buildings. This may not change the overall energy use much, but will create a deviation between the temporal behaviour of the archetype building and the original buildings, as the badly insulated building already starts heating while the lower set-point is still active.

Patteeuw and Helsen (2014) already proposed a method to obtain such a representative aggregated stochastically generated occupant behaviour profile, but they did not compare nor benchmark this method. Moreover, they considered only one particular building envelope model combined with different stochastically generated occupant behaviour profiles. Additionally, their method requires building-by-building simulations or optimizations, which is not in line with the idea of archetype simulations.

This paper studies the aggregation of stochastically generated set-point temperature profiles into representative set-point temperature profiles for archetype buildings, considering two existing residential districts and their variability in building envelope. There is also a short study of the influence of missing occupant data. The research questions are the following:

- How can stochastically generated occupant behaviour profiles be aggregated for archetype simulations? ${ }^{1}$

- How can the computational effort required to aggregate stochastically generated occupant behaviour profiles be limited, without introducing large errors?

- How large is the resulting percentage error on the simulated energy demand by aggregating buildings and their stochastically generated occupant behaviour profiles into archetypes?

- How accurate is the aggregation if only a fraction of the occupant behaviour profiles in the district is known? 

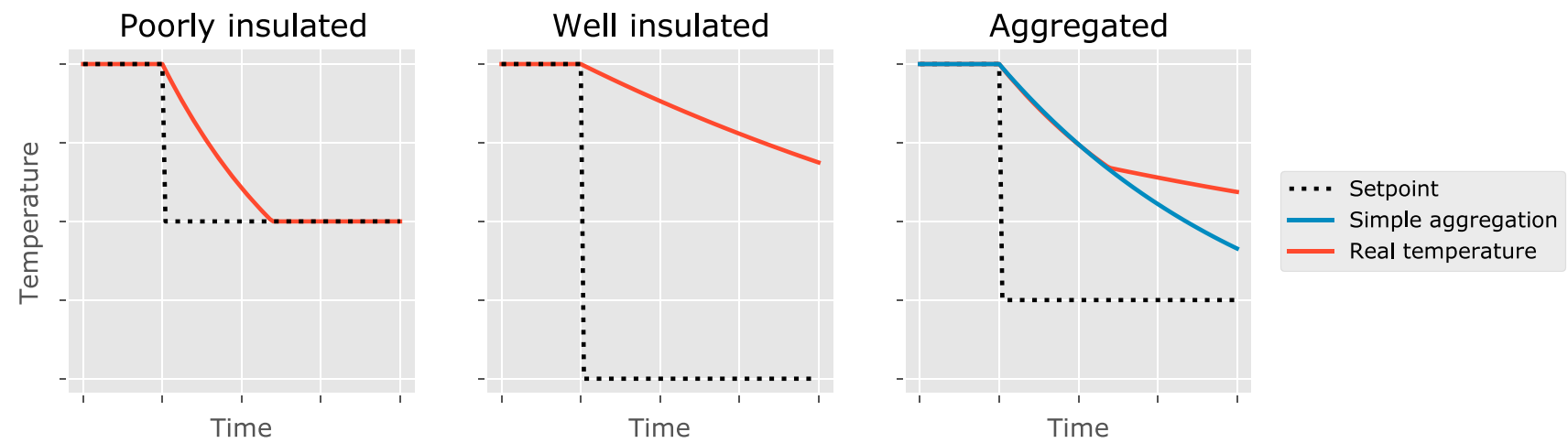

Figure 1. This figure illustrates one shortcoming of a simple averaging of set-point temperatures. In the left graph, the set-point temperature and actual temperature profile of a poorly insulated building are shown. The middle figure shows the same, but for another, well insulated building with a different set-point temperature. The right graph shows the aggregation of the two, the dotted line is the simple average of both set-point temperatures, the solid line at the top is the average of the two temperature profiles, and the solid line at the bottom is the result of a simulation of the archetype building with the average set-point temperature.

In this study, focus is on two residential districts and their energy demand for space heating. Therefore, the considered aspects of the occupant behaviour are the set-point temperatures and the internal heat gains, originating from the occupants, appliances and lighting. The electrical demand of electrical appliances and the heat demand of domestic hot water are not considered, nor is the occupant behaviour regarding window opening and ventilation.

Three methods to obtain a representative occupant behaviour profile for the archetype building are compared. All methods simply average the internal heat gains but embody a different approach to get the representative set-point temperature profile for the archetype buildings. The first method simply calculates the mean of the set-point temperature profiles and serves to show the business-as-usual, whereas the second method determines the actual temperature profiles for all buildings through building-by-building optimizations, yielding the highest accuracy. This method is taken from Patteeuw and Helsen (2014) and serves as a benchmark, as it is a computationally expensive, yet accurate aggregation method. A third method is proposed in this work to reduce the computation load of the second method and to limit the loss in accuracy of the first method.

Answers to the research questions are sought by extensive evaluations of the aggregation error on both the temporal behaviour of and the annual energy demand for space heating. The aggregation error of the three occupant aggregation methods is assessed by comparing their performance to the full simulation/control optimization (i.e. building-by-building). ${ }^{2}$ In addition to the stochastic occupant behaviour, the three methods are also evaluated for deterministic occupant behaviour, excluding the natural variability in occupant behaviour, allowing to assess the error of representing the two districts by six archetype buildings instead of all 847 buildings. Finally, this study is carried out for two building models with a different level of detail to investigate whether the aggregation errors depend on the employed simulation model.

In the next Section, the studied occupant models and the three aggregation methods are introduced first. Then, the case study is presented, along with the considered building models. Their performance is elaborately discussed in Section 3. Finally, the main conclusions are formulated in Section 4.

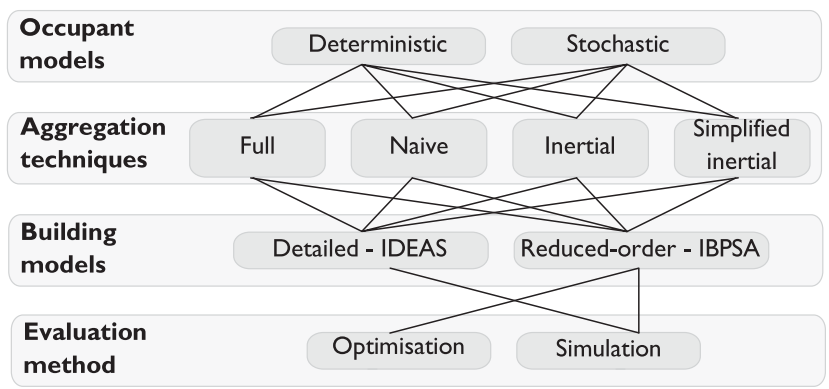

Figure 2. Schematic overview of all combinations that are considered in this work represented by the black lines. Two methods are used to evaluate two building models, combined with two occupant models. Three occupant aggregated methods are considered and compared to their respective full simulation/optimisation.

\section{Method}

In this section, a short overview of the method is given first. Then, both the deterministic and the stochastic occupant behaviour models are explained in Section 2.1. Subsequently, the three different methods to compute the aggregated minimum comfort temperature profile are presented in Section 2.2. The case study is introduced in Section 2.3. Finally, the generation of the building energy models for the full simulation and the archetype simulation is described in Section 2.4, along with the employed simulation/optimization environments.

To investigate how stochastically generated occupant behaviour profiles can be aggregated, three different methods to compute the aggregated set-point temperature profile are developed. To enable an in-depth comparison of the different aggregation methods, they are tested for two different occupant models, two different building energy models and two different evaluation strategies, as presented in Figure 2.

Both a stochastic and a deterministic occupant model are considered. Including the deterministic occupant model allows to illustrate the error caused by solely aggregating the building geometry rather than by aggregating both the building geometry and the occupant behaviour. In addition, three different aggregation techniques are studied. The three different methods are summarized in Table 1 . The naive method simply averages the indoor set-points, whereas the inertial methods include the thermal inertia of the dwelling. In Figure 2, a fourth 
Table 1. An overview of the different computation methods of the aggregated minimum comfort temperature profiles that are considered in this work.

\begin{tabular}{lc}
\hline Name & Description \\
\hline Naive method & $\sum_{i=1}^{n} T_{\text {min, comfort } / n}$ \\
Inertial method & $\sum_{i=1}^{n} T_{\min , i / n}$ \\
Simplified inertial method & $\sum_{i=1}^{n} T_{\min , s i / n}$ \\
\hline
\end{tabular}

technique is included, more in particular the full case (i.e. the building-by-building simulation or optimization), which serves as a reference for the naive, inertial and simplified inertial methods. Furthermore, two different types of building energy models (detailed IDEAS models vs. reduced-order IBPSA models) are compared. As evaluation methods, both simulation and optimization are considered. The simulation and optimization correspond to two types of control for the ideal heating system (PI control vs. optimal control) and are carried out in two different software environments, as introduced below. In the simulation case, the two types of building energy models are compared, yet in the optimization case only the reduced-order models are used to minimize computational efforts. The general work-flow of the full simulations and the archetype simulations is illustrated in Figure 3.

Finally, three different aggregation techniques are studied. The three different methods are summarized in Table 1. The naive method simply averages the indoor setpoints, whereas the inertial methods include the thermal inertia of the dwelling. In

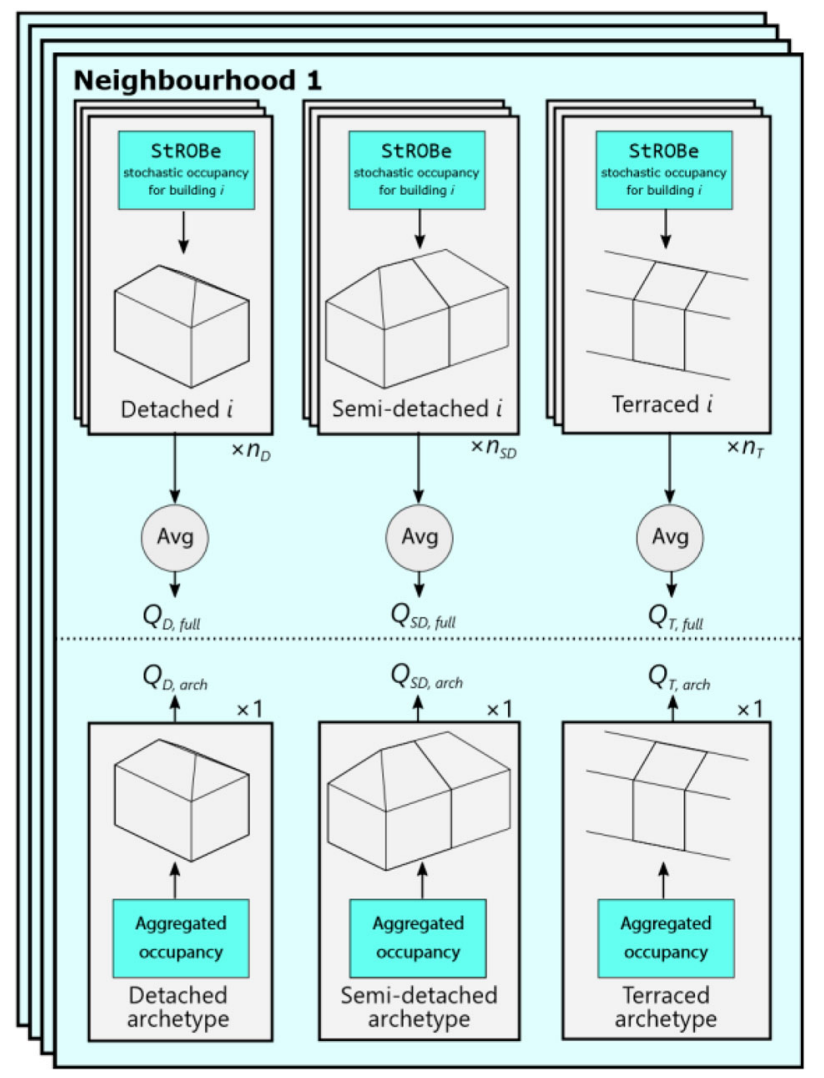

Figure 3. Schematic overview of the aggregation methods described in this paper. The upper half shows the calculation of the full simulations and optimisations, while the lower half shows how the behaviour of all buildings is represented in three archetypes.
Figure 2, a fourth technique is included, more in particular the full case (i.e. the building-by-building simulation or optimization), which serves as a reference for the naive, inertial and simplified inertial methods. The general work-flow of the full simulations and the archetype simulations is illustrated in Figure 3.

In short, for every possible combination of occupant model, building model and evaluation method, the performance of the archetype model with the aggregated set-point temperature profile is compared to the full model for the three aggregation methods. The differences in both the annual energy demand for space heating $\left(E_{S H}\right)$ and the load duration curves (LDC) are assessed for the two neighbourhoods, visualizing amongst others the peak load. The load duration curve is the function that describes for which the number of hours a certain minimum heating power level is exceeded during the year. The LDC is constructed by ordering the values of the yearly heating demand time series in descending order. As a result, the LDC gives a first indication of the temporal behaviour, but does not fully represent the thermal behaviour, as time-dependent profiles do.

\subsection{Occupant behaviour}

The focus of this work is on aggregating single set-point temperature profiles into archetype set-point temperature profiles. The occupant-related parameters considered in this work are internal heat gains, split between convective and radiative gains, and the desired set-point temperature. As the main interest of this paper is on the energy demand for space heating, the mass flow rate for domestic hot water is not considered. Window opening is not considered either.

The deterministic occupant model is constructed according to the ISO 13790 standard (Roulet 2002), which results in an occupant behaviour with a fixed time schedule. The stochastic occupant model is constructed using the StROBe toolbox (Baetens and Saelens 2016). Both models are described in Sections 2.1.1 and 2.1.2.

\subsubsection{Deterministic occupant behaviour: ISO 13790}

The ISO 13790 standard (Roulet 2002) uses a fixed time schedule with constant internal gains. Also, fixed set-point temperatures are set according to this schedule. This means all buildings behave identically, as summarized in Table 2.

In most other studies that employ a deterministic occupant model, the occupant of the archetype building is identical to the occupant of the separate buildings. However, in this study, the three studied aggregation methods are first applied to the deterministic set-point temperature profiles to account for the building thermal inertia.

\subsubsection{Stochastic occupant behaviour: StROBe}

The StROBe Python toolbox (Baetens and Saelens 2016) provides functions to generate stochastic occupant behaviour profiles for

Table 2. Set-points and internal gains (according to the ISO 13790 standard) per unit area as a function of time.

\begin{tabular}{lcc}
\hline Time & Set-point $\left[{ }^{\circ} \mathrm{C}\right]$ & Internal gains $\left[\mathrm{W} / \mathrm{m}^{2}\right]$ \\
\hline 7:00-17:00 & 16 & 8 \\
17:00-23:00 & 21 & 20 \\
$23: 00-7: 00$ & 18 & 2 \\
\hline
\end{tabular}


residential dwellings. Based on the household size, a random household composition is sampled from the database of the Belgian Time-Use Survey from 2005. The household composition defines the occupation of the individual household members, i.e. 'minor', 'full-time employed', 'part-time employed', 'unemployed' or 'retired'. Then, the occupancy of each household member, i.e. 'awake at home', 'asleep at home' or 'absent', is estimated, based on a heterogeneous discrete-time Markov chain. Subsequently, their activities are predicted, resulting in the different stochastically generated occupant behaviour profiles on household level. These profiles include the mass flow rate of DHW tapping, the internal heat gains (separate convective and radiative gains), the use of electrical appliances and the setpoints temperature for different zones in a building (day zone, night zone and bathroom). Each profile consists of 52 identical weeks. For more information, the reader is referred to the work of Baetens and Saelens (2016). They also provide insight into the resulting variability between the different StROBe profiles.

In this study, the distribution of the household sizes in the studied district is identical to the distribution of the household sizes for Belgium. The household size for each dwelling is sampled randomly from this national distribution. It is also assumed that data on the employment type is available, resulting in a tailor-made stochastically generated occupant behaviour profile for each dwelling. As a result, a fixed StROBe profile is assigned to each building in the two neighbourhoods, eliminating the effect of the randomized occupant profiles between consecutive simulations.

\subsection{Aggregation of occupant behaviour}

This paper discusses three ways to aggregate stochastically generated occupant behaviour profiles. The aggregation is needed to construct a occupant behaviour profile for the archetype building, representing the behaviour of all buildings belonging to that archetype. The aggregation is obtained by averaging the different profiles of the occupant behaviour (i.e. the mass flow rate of DHW tapping, the internal heat gains, the use of electrical appliances and the set-point temperatures). However, as already demonstrated in Figure 1, a simple aggregation of the set-point temperature profiles can lead to serious errors in the district temporal behaviour.

Three different methods to average the set-point temperature profiles are proposed: the naive, inertial and simplified inertial methods, all of which are described in Sections 2.2.1, 2.2.2 and 2.2.3 respectively. Each uses a different method to calculate the minimum set-point temperature profiles of all buildings within the two neighbourhoods, after which these profiles are aggregated for each of the archetype buildings.

\subsubsection{Naive aggregation method}

The simplest way to construct an aggregated set-point temperature profile for all buildings that belong to one particular archetype building is to average their contribution to a single profile. The naive aggregation in the deterministic case implies that the aggregated occupant is identical to the occupant of the single buildings. The naive aggregation in the stochastic case is illustrated for two buildings in Figure 1.

However, this way of aggregation naively neglects that the actual temperature in the buildings will differ from their respective set-points, as a result of thermal inertia of the buildings. As a result, this way of aggregation is referred to as the naive aggregation method, to distinguish it from the two alternative methods.

\subsubsection{Inertial aggregation method}

This method was proposed by Patteeuw and Helsen (2014), who used it to aggregate the behaviour of identical building models with different occupant behaviour. The present work studies whether this computationally expensive, yet accurate aggregation method is valid for the aggregation of different building models as well.

The aggregated set-point temperature profile for the archetype building is calculated by solving an optimal control problem of the heating system of each separate building in the cluster. Note that although each building has to be simulated separately, this method still entails a simplification, as it removes the possibility to let buildings within a district interact with one another. While this method uses optimization, simulation could be used instead. As opposed to simulation, optimization causes the building to pre-heat and to maintain a constant indoor temperature once the set-point temperature is reached. As the ideal heating system model assumes radiators to be present in the dwellings, the heat-up time of the dwellings is assumed to be low, reducing the difference between simulation and optimization. Optimization was preferred in this work with future research activities in mind. These activities focus on the optimal design and control of district heating networks.

The proposed methodology finds the optimal space heating radiator control to minimize the energy use while still respecting the comfort boundaries, and this for each building with its own StROBe profile. Aggregating these optimal profiles will result in a more gradually changing profile that takes the effects specific to each building into account. For example, this methodology would produce the red curve in Figure 1, as it can detect that the poorly insulated building has to start heating earlier to maintain the lower set-point.

The formulation of the optimization problem for a single building is shown in Equation (1). In this model, $\dot{Q}_{t}$ is the thermal power delivered to the building during time step $\Delta t$. There are $n$ time steps, which together form the set T. The temperatures within the building $\tilde{T}_{t}$ are determined by the IBPSA RCmodel $f_{I B P S A}$ (Section 2.4.1), the historic building temperatures, the external inputs, such as the weather $u_{t}$, and the length of the time step $\Delta t$. An important constraint in this problem is the comfort constraint, that requires temperatures to be above a minimum temperature $T_{\min , c o m f o r t}$. As the objective is a minimization of energy, the control will always try to keep building temperatures as low as possible. Hence, the maximum comfort temperature is of less importance in this case. However, it is possible to violate this constraints, if the heating system is unable to keep the temperature between the comfort bounds. This is done by use of the slack variable $D_{t}$. This value offsets the constraint when the model would otherwise become infeasible, and is penalized in the objective function with a very large weight (i.e. a large cost) $c$. This is mostly necessary to allow overheating due to solar and internal gains during mid-season and summer months, as there is no cooling system. This optimization problem in Equation (1) is written and solved with the use of modesto, which is introduced in section 2.4.3. Averaging all optimized 
temperature profiles leads to the aggregated minimum comfort temperature for the archetype building.

$$
\begin{array}{cll}
\underset{\dot{Q}_{t}}{\operatorname{minimize}} & \sum_{t=1}^{n}\left(\dot{\mathrm{Q}}_{t} \Delta t+{ }_{c} D_{t}\right) & \\
\text { subject to } & \vec{T}_{t+1}=f_{I B P S A}\left(\vec{T}_{t}, \vec{u}_{t}, \dot{Q}_{t}, \Delta t\right) & \forall t \in T \\
& T_{\text {min,comfort } t} \leq T_{\text {zone }, t}-D t & \forall t \in T
\end{array}
$$

However, in this method, overheating should be treated with special care. During summer, due to the higher outdoor temperature, the solar gains and the internal heat gains, and especially because no cooling system nor window opening were considered, the building temperature exceeds the set-point, which leads to overheating of the buildings. Overheating causes the aggregated set-point temperature of the inertial method to rise above the maximum comfort temperature from the stochastically generated occupant behaviour (called inertial aggregation method with overheating). When this aggregated temperature profile is considered in the archetype simulation or optimization, the set-point temperature exceeds the room temperature that would be achieved by the archetype model in a free-floating scenario, ${ }^{3}$ hence causing the building to use heat during this period, leading to an overestimation of the $E_{S H}$.

In order to eliminate this problem of overheating the archetype, ${ }^{4}$ the room temperature of each individual building is limited to the maximum set-point temperature of its particular StROBe profile, which is done after optimization of the individual buildings and before averaging all individual optimized temperature profiles. As such, the inertial aggregation method without overheating is obtained, which is referred to as the inertial aggregation method in the remainder of the text.

\subsubsection{Simplified inertial aggregation method}

The goal of using an advanced method, such as the inertial method, is to include the thermal inertia of the building and to obtain a more representative set-point temperature profile for the archetype building. However, in the inertial method, the thermal inertia is incorporated in the aggregated minimum temperature profile through a full-year optimization of the energy use of the building heating system. This full-year optimization of every building in the two neighbourhoods is computationally expensive. To limit these computation time and efforts, a simpler method has been developed, which will be referred to as the simplified inertial aggregation method.

The simplified inertial method is based on the fact that a simple pattern can be found in the minimum temperature in a building, as can be seen in Figure 4 . When the requested comfort temperature is high, the temperature is maintained as good as possible by the heating system. When the comfort temperature decreases (e.g. during the night), the temperature decreases following a first-order model, being an exponential decay. The simplified inertial method assumes this behaviour is always present. Hence, during moments of high temperature demands, the resulting minimum temperature equals this high temperature. As soon as the set-point temperature decreases, the exponential decrease in temperature is calculated with a one-step-ahead simulation of the reduced-order IBPSA models (Section 2.4.1), as is shown in Equation (2) and Figure 4.

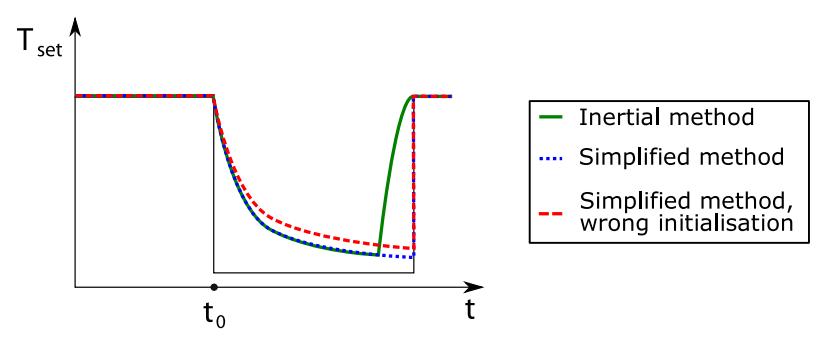

Figure 4. An illustration of the simplified inertial method principle. The black thin solid line shows the lower comfort boundary in a building, the green thicker solid line is the temperature calculated by the inertial method. The blue dotted line shows the simplified inertial method, if the initial conditions of the exponential decrease are known. The red dashed line shows the results of the simplified method, if the initial conditions have been estimated.

In this equation, the temperatures of all states in the model at time $t+1, \tilde{T}_{t+1}$, are calculated by using the reduced-order IBPSA models, the temperatures in the previous time step $\tilde{T}_{t}$, the inputs $\sim u_{t}$, such as the outside temperature, solar and internal gains, the thermal power injected into the building $\dot{Q}_{t}$, and the time step $\Delta t$ between two points in the time. Note that in the simplified inertial method, $Q_{t}$ is always zero, as only the cool-down of a building is calculated.

Additionally, this simplified method assumes the heating of the building happens instantly, as is shown in Figure 4. We assume the power of the heating system is sufficiently high such that the time constant of a temperature increase is negligible with respect to that of a temperature decrease. This assumption is not valid for floor heating systems. As a result, the deviations between the archetype simulation/optimization and the full simulation/optimization would be larger when the building is equipped with slowly-reacting emission systems (such as floor heating).

$$
\vec{T}_{t+1}=f_{I B P S A}\left(\vec{T}_{t}, \vec{u}_{t}, \dot{Q}_{t}, \Delta t\right)
$$

At the start of a temperature drop in the building ( $t_{0}$ in Figure 4 ), an assumption of the initial conditions is required. A different initialization can lead to a different temperature trajectory in the heated zone, as shown in Figure 4. For the reduced-order IBPSA models, used in this work, initial conditions are required for the model nodes related to the building air, the internal and external walls, the roof and the floor. The building air temperature is assumed equal to the comfort temperature before the drop. Through simulation, it appeared that the internal walls have a temperature close to the building air temperature, while the external walls have a temperature close to the outside temperature. For the initial external wall temperature, a weighted average between the outside and building air temperature was found to give the best results. The roof temperature is usually close to the external temperature, although it also highly depends on the solar radiation. Nonetheless, we assume the initial roof wall temperature to be equal to the outside temperature. The floor temperature appears to be close to the ground temperature, as such, we initialize it with the ground temperature at the start of a temperature drop.

This simplified method has similar overheating problems as the inertial method and deals with these problems in an identical way. The simplified inertial aggregation method with overheating is not shown in this work due to its worse performance, 


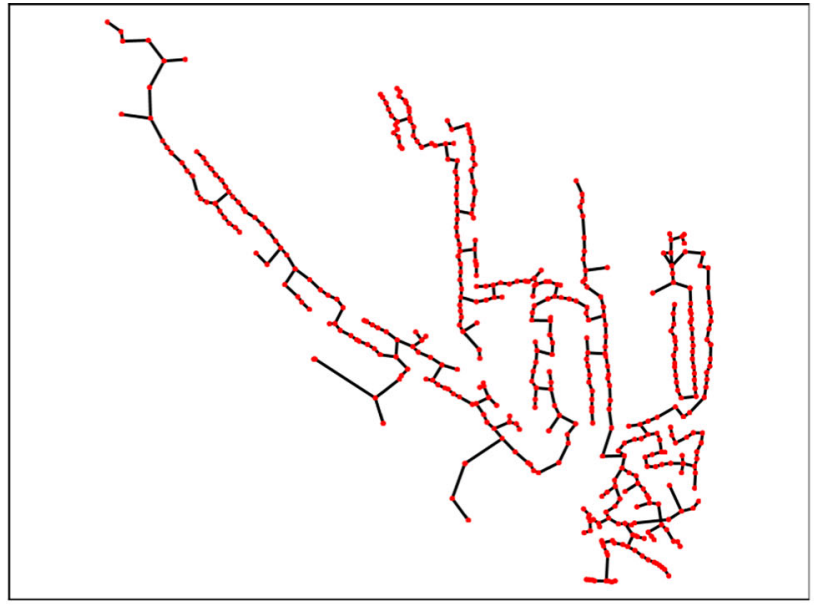

(a) Oud-Winterslag

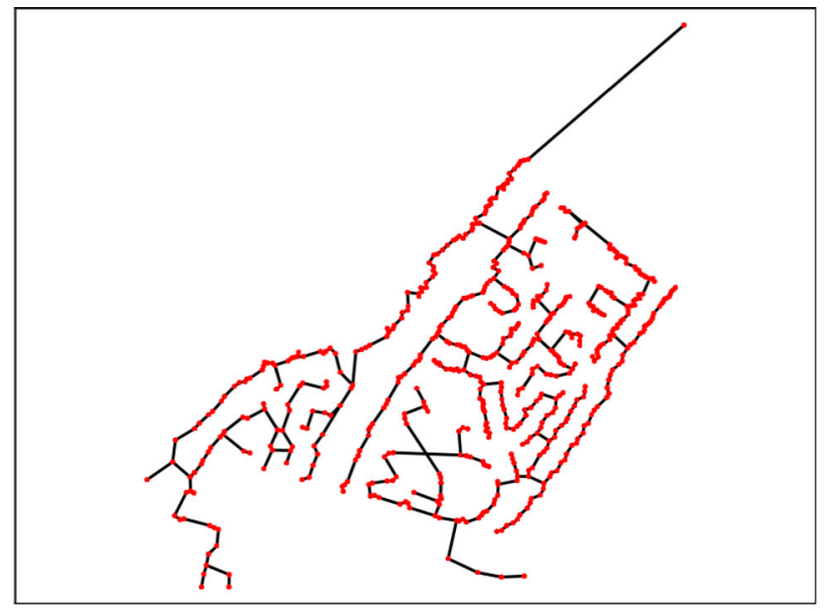

(b) The north-east of Zwartberg

Figure 5. The two investigated neighbourhoods. The dots indicate the position of the buildings, the lines indicate the streets.

as shown later in Section 3 for the inertial method. As a result, the simplified inertial aggregation method refers to the simplified inertial aggregation method without overheating.

\subsection{Case study description}

Two neighbourhoods are selected to investigate the influence of occupant behaviour aggregation on the district heat demand for space heating. These two neighbourhoods, Oud-Winterslag and the north-east part of Zwartberg, are both situated in Genk, a city in the north-east of Belgium. These neighbourhoods were selected because a CityGML model with the level of detail (LOD) 2 (Kolbe, Gröger, and Plümer 2005) is available for these neighbourhoods, enabling more detailed energy simulations. Oud-Winterslag and the north-east of Zwartberg consist of 350 and 497 buildings respectively and are shown in Figure 5 .

While Oud-Winterslag is a relatively new neighbourhood (with most buildings built in the 2000s), Zwartberg is an older neighbourhood, dating from the 1950s. As a consequence, the dwellings in Zwartberg are less insulated and demand more heat to maintain the desired indoor climate. This gives an opportunity to test the aggregation methods on buildings with different insulation standards.

Additionally, it is assessed what would happen if only a limited amount of information is available, i.e. not all occupant behaviour profiles. This allows to test the influence of an incomplete data set. To do so, the detached houses in the largest district, namely Winterslag, are studied. The aggregated setpoint temperature profile for the Winterslag detached archetype building is calculated using only 1, 5, 10, 25, 50, 100 or 200 profiles, randomly sampled out of the 291 buildings that are represented by this archetype building. For each of these cases, the simplified inertial aggregation method is applied to the corresponding number of set-point temperature profiles. The random selection of $n$ set-point temperature profiles is repeated 10 times to investigate the influence of the random set-point temperature profile selection. The results of this exercise are shown in Section 3.2.4.

\subsection{Buildings}

To automatically generate the building energy models for OudWinterslag and the north-east part of Zwartberg, TEASER, a Python package developed at RWTH Aachen (Remmen et al. 2018), is used, though in an adaptation as described by De Jaeger et al. (2018b). To perform the full simulation as well as the archetype simulations, both detailed IDEAS models and reduced-order IBPSA models available in the IDEAS Modelica library (OpenIDEAS 2019) are used and simulated in Dymola (Dassault Systèmes AB 2018) (Table 2). Additionally, the reducedorder IBPSA models are used for optimization in modesto as well (Table 2). These software packages are now described briefly, along with the archetype calculation.

\subsubsection{Building models}

The adapted TEASER package exports both highly detailed IDEAS Modelica building models and reduced-order IBPSA models, based on an LOD2 CityGML model containing building geometry, building function, construction year, number of storeys and storey height. However, the available CityGML model only contains geometry-related information and therefore the construction year has to be added manually. Based upon a Google Streetview survey, all buildings of one neighbourhood are assumed to share the same construction year due to the homogeneous character of both neighbourhoods (Table 2).

To export the building models and perform dynamic energy simulations, additional thermal properties are required and allocated by TEASER based on the construction year. The thermal properties are based on statistical information and are derived from the TABULA project for Belgium (Cuypers et al. 2014). The exact assumptions, as well as additional features that were added to TEASER, are given in (Protopapadaki, Reynders, and Saelens 2014). The infiltration rate is set to $0.4 \mathrm{ACH}$ at real conditions for all models, corresponding to $8 \mathrm{ACH} @ 50 \mathrm{~Pa}$. Also, the building geometry is simplified by merging the walls, windows and roofs for each of the four orientations (north, east, south and west) to speed up the simulation with a negligible loss of accuracy (De Jaeger et al. 2018b). 
Table 3. An overview of the different archetype models under consideration.

\begin{tabular}{|c|c|c|c|c|c|}
\hline Abbreviation & Constr. type & Neighbourhood & Constr. year & Number of buildings & Average U-value $\left(\mathrm{W} /\left(\mathrm{m}^{2} \mathrm{~K}\right)\right)$ \\
\hline Winterslag D & detached & Oud-Winterslag & 2000 & 291 & 0.86 \\
\hline Winterslag SD & semi-detached & Oud-Winterslag & 2000 & 53 & 0.86 \\
\hline Winterslag T & terraced & Oud-Winterslag & 2000 & 6 & 0.86 \\
\hline Zwartberg SD & semi-detached & North-East Zwartberg & 1950 & 209 & 2.13 \\
\hline Zwartberg T & terraced & North-East Zwartberg & 1950 & 156 & 2.13 \\
\hline
\end{tabular}

After enriching the building geometry with the building envelope characteristics, the models are automatically generated and simulated in the IDEAS Modelica library. The Integrated District Energy Assessment Simulations (IDEAS) library (Jorissen et al. 2018) is implemented in the Modelica language (Modelica Association 2017) and allows simultaneous transient simulation of thermal, control and electric systems at both building and district level.

In the detailed IDEAS models, all building envelope components are fully characterized in terms of their layers and materials. The transient thermal phenomena within the building are modelled using a zonal modelling approach, assuming perfect mixture of the air inside the zone. This building model is described in (Jorissen et al. 2018). In the reduced-order IBPSA models, the building envelope components are modelled as resistance-capacitance $(\mathrm{R}-\mathrm{C})$ combinations. A four element model is used, corresponding to four $\mathrm{R}-\mathrm{C}$ combinations of which one for all exterior walls, one for the roof, one for the ground floor and one for the interior walls and floors. All resistance and capacitance values are calculated within TEASER (Remmen et al. 2018). This building model is described more elaborately in (Lauster et al. 2014). Both building models use one thermal zone for the whole building.

Subsequently, an ideal heating system is linked to these building envelope models, but the exact implementation depends on whether the simulation or optimization environment is considered. Therefore, the ideal heating system is discussed together with these environments in Section 2.4.3. The buildings are assumed not to have a ventilation system, as this is not common considering the age of the dwellings. All simulations/optimizations in this study are conducted for the heating dominated climate of Uccle (Belgium).

\subsubsection{Archetype calculation}

To reduce the computational burden caused by the full simulation, district energy simulations are often based on archetypes. How to aggregate a particular district into a limited number of archetypes has been the focus of a number of studies (Ghiassi and Mahdavi 2017; Tardioli et al. 2018). These methods often employ rather complex clustering techniques. As the focus of this work is on aggregating the occupant behaviour rather than the building envelope, a simplified method to create the archetype building definitions was used in this work and is introduced here.

Each of the neighbourhoods was aggregated into three archetype buildings, following their building type, i.e. terraced, semi-detached or detached dwelling, resulting in six archetypes for the two neighbourhoods. An overview is given in Table 3. As all buildings of each neighbourhood were assumed to share the same construction year, they have identical compositions for their building envelope components since all building envelope characteristics were allocated based on this construction year. As a result, only the building geometry needs to be aggregated, which is achieved by averaging all geometrical characteristics (i.e. heated volume, heated floor area, ground floor area, exterior wall area, window area, roof area, interior wall area and interior floor area). Since all walls, windows and roofs are merged towards four orientations, calculating the average is quite straightforward. As a result, the archetype buildings used in this work are non-existing average buildings instead of real sample buildings.

\subsubsection{Building simulation and optimization}

The detailed IDEAS models are simulated in Dymola, whereas the reduced-order IBPSA models are both simulated in Dymola and optimized in modesto (Vandermeulen et al. 2018). The exact simulation/optimization strategies within Dymola and modesto are explained below.

In Dymola, both the detailed IDEAS models and reducedorder IBPSA models for the building envelope are linked to an ideal heating system model. This heating system model only delivers hot water for space heating. It does not include domestic hot water (DHW). The heating system model is the Ideal Radiator Heating model found in IDEAS 1.0.0 (Baetens et al. 2015). In this model, the heat source has $100 \%$ efficiency and perfect modulation. Its maximum power is limited by the building design power. The design power is based on a steady-state calculation of that building with an outside temperature of $-8^{\circ} \mathrm{C}$, a ground temperature of $10^{\circ} \mathrm{C}$ and an indoor temperature of $21^{\circ} \mathrm{C}$. This heat source is directly connected to the radiator, in which the heat transferred to the building is split into two parts: radiative heat transfer and convective heat transfer. In this radiator model, this fraction is constant and set to $30 \%$ radiative and $70 \%$ convective. The models are simulated in Dymola using the Dassl solver with an output interval of $10 \mathrm{~min}$. The simulations are conducted for a period of 1 year, with an additional initialization period of 1 month.

In modesto, optimal control is performed using the problem described in Section 2.2.2. The optimizations span one year, and an additional week is used for the initialization of the model states at the start of the optimization.

\section{Results}

Different methods to aggregate stochastically generated setpoint temperature profiles are proposed in this work. To compare the different methods and assess their performance, multiple simulation/optimization strategies have been considered, as described in Section 2 and illustrated in Figure 2. Their results 

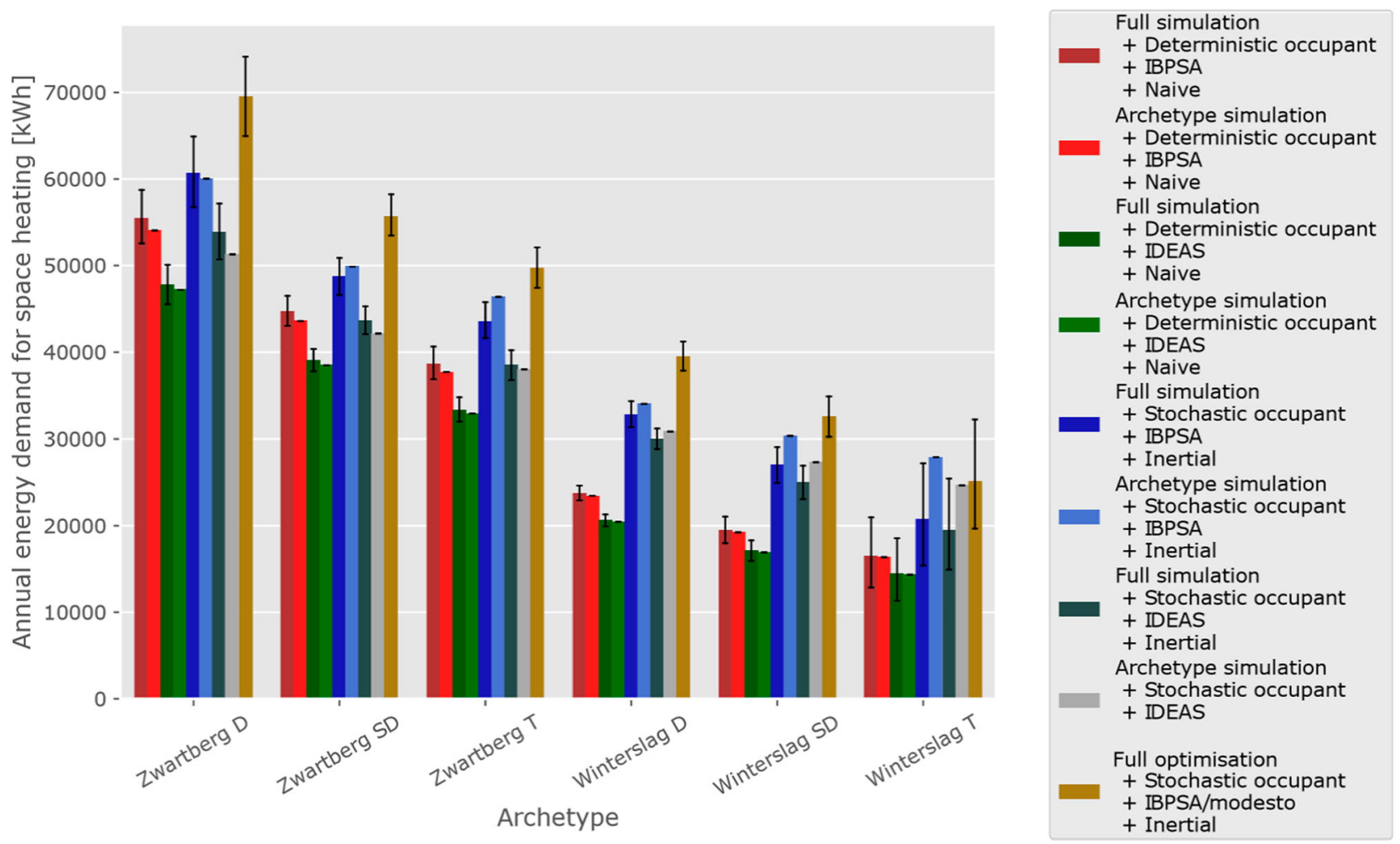

Figure 6. Comparison of the average annual energy demand for space heating $\left(E_{S H}\right)$ for the six archetype buildings for the different simulation/optimisation strategies. The Full simulation is the result of all buildings simulated separately. The archetype simulation shows the result of the naive and inertial aggregation method for the deterministic and stochastic models, respectively. The height of the bars represents the average of all buildings that follow this archetype building. The error bars indicate

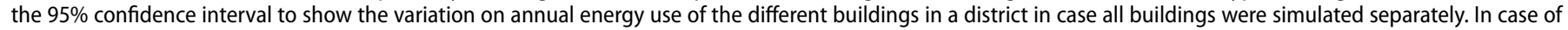
the archetype simulation there is only one value and hence no confidence interval.

are presented in this Section. However, not all possible combinations displayed in Figure 2 are discussed. Especially concerning the deterministic occupant behaviour, only those combinations that contain new and relevant insights concerning the aggregation errors are presented. First, the archetype simulation is compared to the full simulation for the deterministic occupant behaviour, by assessing both $E_{S H}$ and LDC of the district. Subsequently, aggregation of the stochastically generated occupant behaviour is considered, based on the $E_{S H}, L D C$ and the computation times. Finally, the performance of considering a limited number of profiles to aggregate the stochastically generated occupant behaviour is assessed.

\subsection{Aggregating deterministic occupant behaviour}

To assess the impact of aggregating the district into six archetype buildings, three per neighbourhood, without considering a variability in occupant behaviour, the full simulations are compared to the archetype simulations through the use of deterministic occupant behaviour. Although the deterministic archetype occupant can also be aggregated in three different ways, only the naive method and the simplified inertial method (without overheating) are presented in this work.

Figure 6 illustrates the average $E_{S H}$ of the deterministic full and archetype simulations for the reduced-order IBPSA models and the detailed IDEAS models, in red and green respectively, while using the naive aggregation technique for the archetype simulations. Figure 6 also shows the results for the stochastically generated occupant behaviour, which will be explained in Section 3.2. In addition, Table 4 summarizes these results numerically.

For the naive aggregation technique, the $E_{S H}$ of the deterministic archetype simulations is almost identical to their respective full simulations, as the deviation varies between $-1.19 \%$ and $-2.20 \%$, depending on the considered evaluation method and building model (Table 4). The simplified inertial method performs slightly better than the naive method, although it is only studied for the deterministic occupant while optimizing $E_{S H}$. For the optimized $E_{S H}$, the deviation decreases from $-2.20 \%$ for the naive method to $-1.52 \%$ for the simplified inertial method (Table 4).

Figure 7 shows the LDC of the optimized $E_{S H}$ for the full optimization and the archetype optimization, while using the naive method as well as the simplified inertial method. Overall, these LDCs show a very similar behaviour, even when using the naive method, except in the higher power range. This difference is due to the calculation of the design heat power. These design heat powers are calculated based on the method described in Section 2.4.3, for both the individual buildings and the archetype buildings. As is shown in Table 5, the average design heat power of the individual buildings does not correspond to the design heat power of the archetype building, with mismatches up to $4.8 \mathrm{~kW}$ depending on the archetype, leading to a significant deviation in the peak hours. The results could be improved by a better estimation of the design power of the archetype building. Yet, this is left for future work. 
Table 4. Percentage error of $E_{S H}$ of the different aggregation methods with respect to the full simulation/optimisation case (no aggregation).

\begin{tabular}{|c|c|c|c|c|}
\hline \multirow[b]{3}{*}{ Occupant model } & \multirow[b]{3}{*}{ Aggregation method } & \multicolumn{3}{|c|}{ Evaluation method (\% difference) } \\
\hline & & \multirow{2}{*}{$\begin{array}{c}\text { Optimisation } \\
\text { Reduced-order IBPSA }\end{array}$} & \multicolumn{2}{|c|}{ Simulation } \\
\hline & & & Reduced-order IBPSA & Detailed IDEAS \\
\hline Stochastic & $\begin{array}{l}\text { Full optimisation/simulation } \\
\text { Naive } \\
\text { Inertial } \\
\text { Inertial with overheating } \\
\text { Simplified inertial }\end{array}$ & $\begin{array}{c}41,908 \mathrm{MWh} \\
-7.14 \\
0.22 \\
7.94 \\
3.67\end{array}$ & $\begin{array}{l}36,063 \mathrm{MWh} \\
-8.45 \\
-0.89 \\
3.31 \\
-1.37\end{array}$ & $\begin{array}{l}32,411 \mathrm{MWh} \\
\quad-7.30 \\
-2.85 \\
-1.03 \\
-2.08\end{array}$ \\
\hline
\end{tabular}

Note: The comparison is made for the different occupant models and evaluation methods.
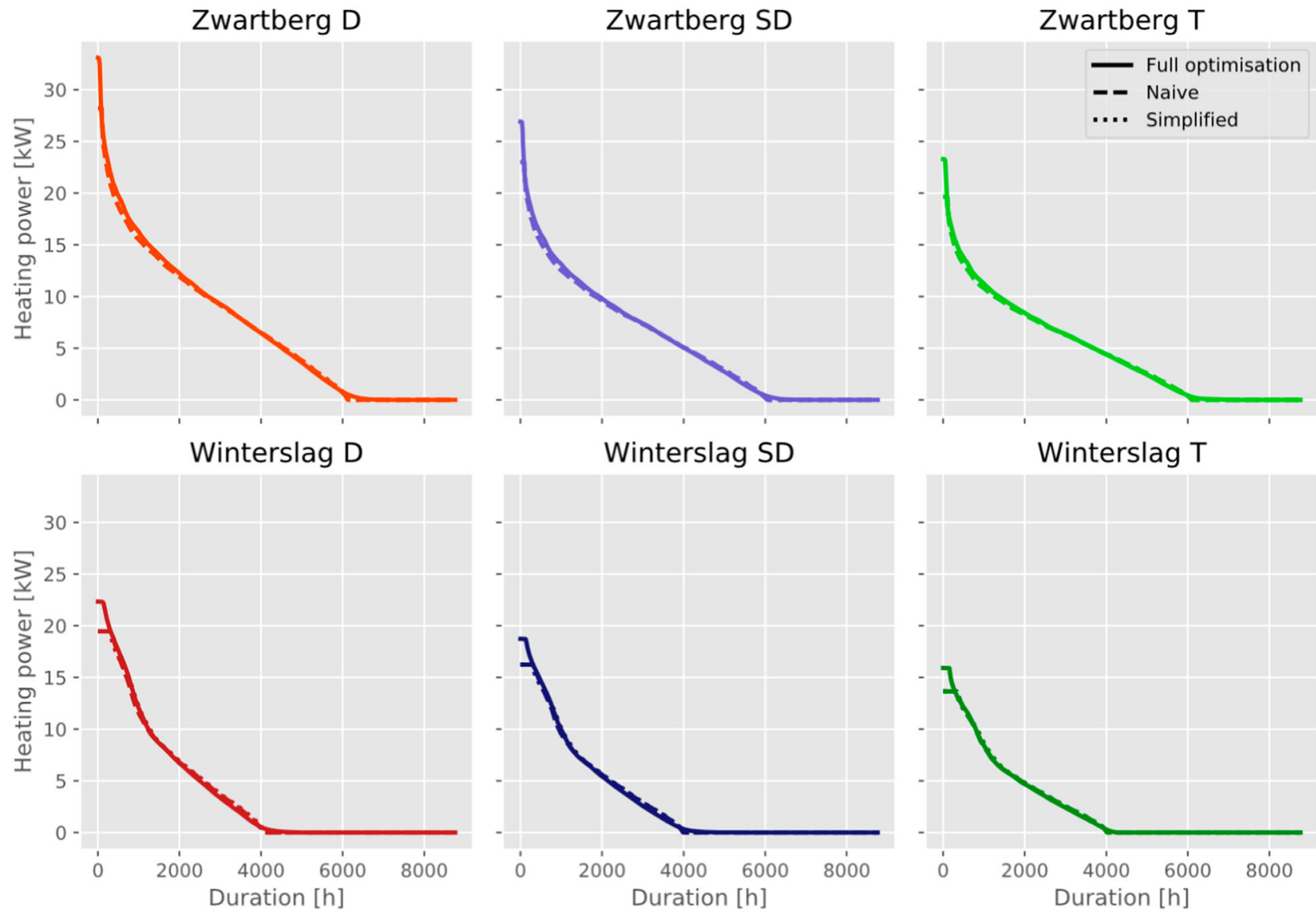

Figure 7. Comparison of the load duration curves for the six archetype buildings and two aggregation techniques with the full optimisation as a reference. Deterministic occupant behaviour profiles are used. Although the results are good at lower powers, the difference in design powers leads to a mismatch in the high power range.

Table 5. A comparison of the average design heat power of the individual buildings (Buildings) and the design heat power of the archetype building (Archetype). All powers are expressed in $\mathrm{kW}$.

\begin{tabular}{lcc}
\hline Cluster & Buildings & Archetype \\
\hline Winterslag D & 22.3 & 19.5 \\
Winterslag SD & 18.7 & 16.2 \\
Winterslag T & 15.9 & 13.6 \\
Zwartberg D & 33.0 & 28.2 \\
Zwartberg SD & 26.9 & 23.0 \\
Zwartberg T & 23.3 & 19.6 \\
\hline
\end{tabular}

\subsection{Aggregating stochastic occupant behaviour}

To assess the impact of aggregating the stochastically generated occupant behaviour, the results of the three studied methods are presented. First, the focus is on $E_{S H}$. Then, the load duration curves are discussed, as they allow to analyse the temporal behaviour of the different methods. When comparing these results to the deterministic profiles of previous Section, it should be kept in mind that the average indoor set-point temperature and internal gains are higher for the stochastic occupant model than for the deterministic occupant model, resulting in higher heating demands. Subsequently, the computation times are discussed. Finally, the performance of considering a limited number of profiles to aggregate the stochastically generated occupant behaviour is assessed. This allows to investigate the effect of an incomplete neighbourhood description on the accuracy of the heat demand simulations. 

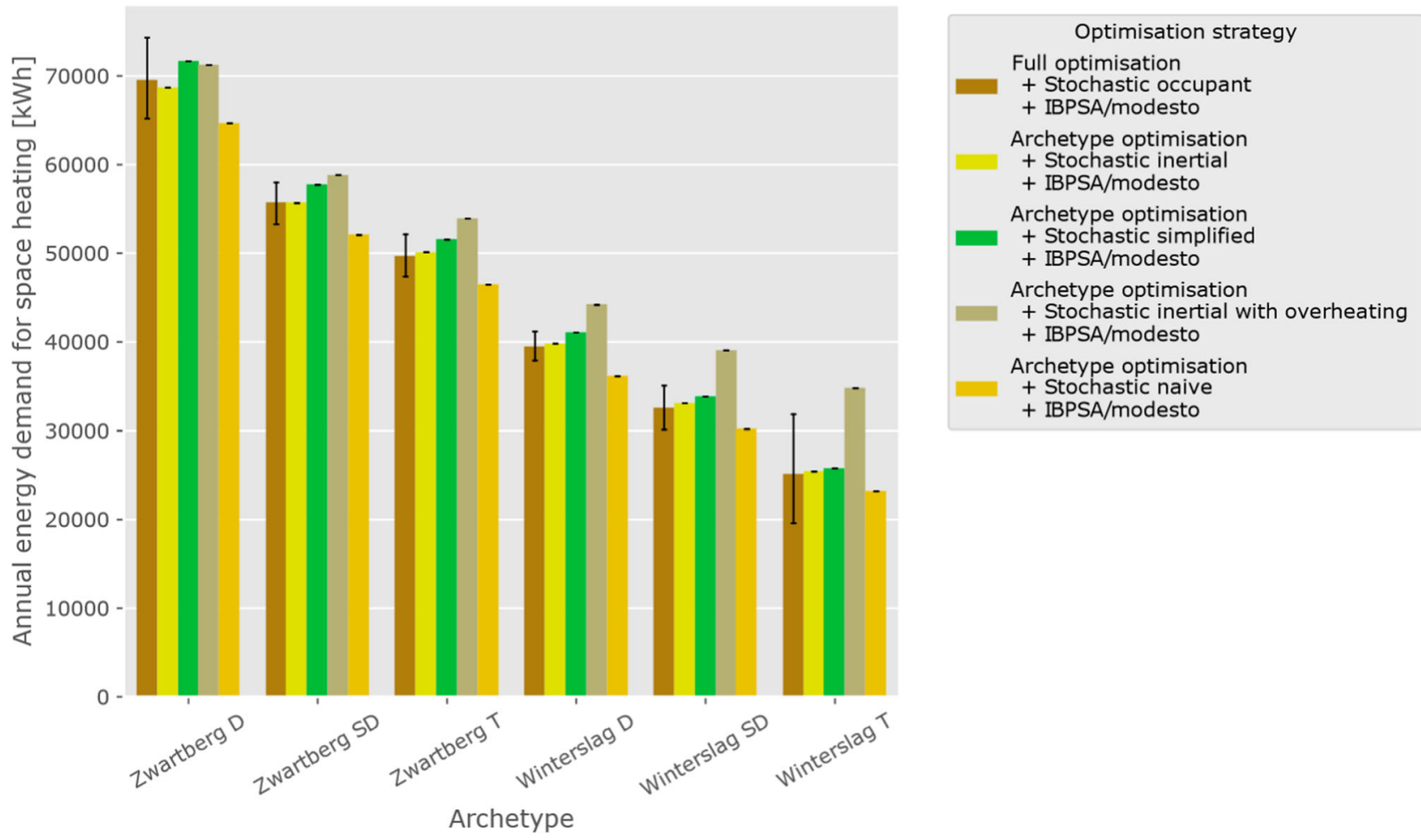

Figure 8. Comparison of the average $E_{S H}$ of the full optimisation in modesto to the different occupant behaviour aggregation methods.

\subsubsection{Annual energy demand for space heating}

First, the performance of the three aggregation techniques is analysed. Figure 8 compares the optimized $E_{S H}$ for the different aggregation techniques of the stochastically generated occupant behaviour for each archetype. These results on district level can also be found in Table 4. Looking at the optimized $E_{S H}$, the inertial aggregation method matches the full optimization most closely, with an error on the $E_{S H}$ of less than $1.6 \%$, depending on the considered archetype. The simplified inertial aggregation method leads to a slightly higher overestimation of the optimized $E_{S H}$ (up to $4.0 \%$ ) compared to the inertial aggregation method and performs second-best for five of the six archetypes.

The naive aggregation method significantly underestimates the $E_{S H}$ (up to $-8.4 \%$ ), whereas the inertial aggregation method with overheating results in an overestimation of the $E_{S H}$ (up to $38.7 \%$ ). Both observations are expected, as discussed in Sections 2.2.1 and 2.2.2. Not accounting for the slower cooling down of the building mass of the represented buildings in one archetype, as the naive method does, leads to an underestimation of $E_{S H}$. Including overheating, on the other hand, causes the aggregated set-point temperature to rise above the maximum comfort temperature, resulting in an overestimation of the district energy demand.

Additionally, this work includes two neighbourhoods with a different thermal performance (the older northeast part of Zwartberg and the newer Oud-Winterslag) enabling the assessment of the three aggregation methods for both older and newer buildings. For the inertial aggregation method with overheating and for the naive aggregation method, the errors of the archetype optimization compared to the full optimization are slightly larger for the newer neighbourhood Winterslag than for the older neighbourhood Zwartberg. The buildings in the newer neighbourhood Winterslag are more prone to overheating due to their increased insulation quality. Therefore, the aggregated set-point temperature profile obtained through the inertial aggregation method with overheating differs more from the demanded set-point temperature profile for Winterslag than for Zwartberg. The buildings in the newer neighbourhood Winterslag also cool down slower, which is not taken into account in the naive aggregation method. The slower the buildings cool down, the worse the naive aggregation methods performs. For the inertial aggregation method and the simplified inertial aggregation method, no significant differences are noted between the two neighbourhoods. Also, no significant differences are noted between the three archetype buildings of 1 particular neighbourhood. However, it is assumed that all buildings within one neighbourhood share the same construction year. As a result, all dwellings that correspond to the same archetype share an identical building envelope definition, except for their geometry.

Although no significant differences in the results of the two neighbourhoods are noticed, more differentiated residential neighbourhoods combined with more advanced archetype definitions should be studied in the future. Nevertheless, it is unlikely that dwellings that follow the same archetype will differ extensively, justifying the assumptions made within this work. In addition, the application to mixed-use districts should be further explored within future work.

All these observations are also valid for the simulated $E_{S H}$, at least if considering the reduced-order IBPSA model. Table 4 shows the percentage differences in $E_{S H}$ for the whole district of the three aggregation techniques for the two evaluation methods, the two-building models and the two occupant models, while using the full simulation/optimization as a reference. 
Table 6. Comparison between annual energy demand for space heating $\left(E_{S H}\right)$ for different evaluation methods and the different building models, using the stochastic occupant model. The full optimisation/simulation was considered to assess only the differences for the various evaluation methods. The reduced-order IBPSA simulation is taken as a reference (ESH in $\mathrm{MWh}$ ), because this model can both be simulated and optimised. The other evaluation methods are compared with their percentage deviation. The deviations are shown on archetype, neighbourhood and district level. The shown ESH for the archetypes is the sum of all dwellings belonging to that archetype.

\begin{tabular}{|c|c|c|c|}
\hline \multirow[b]{2}{*}{ District scale } & \multirow{2}{*}{$\begin{array}{c}\text { Reference (MWh) } \\
\text { Simulation } \\
\text { Reduced-order IBPSA }\end{array}$} & \multicolumn{2}{|c|}{ Evaluation method (\% difference) } \\
\hline & & $\begin{array}{c}\text { Optimisation } \\
\text { Reduced-order IBPSA }\end{array}$ & $\begin{array}{c}\text { Simulation } \\
\text { Detailed IDEAS }\end{array}$ \\
\hline Whole district & 36,063 & 16.2 & -10.1 \\
\hline Zwartberg & 24,975 & 14.3 & -11.0 \\
\hline Oud-Winterslag & 11,088 & 20.5 & -8.2 \\
\hline Zwartberg D & 8005 & 14.6 & -11.3 \\
\hline Zwartberg SD & 10,176 & 14.3 & -10.4 \\
\hline Zwartberg $\mathrm{T}$ & 6794 & 14.0 & -11.6 \\
\hline Winterslag D & 9532 & 20.50 & -8.3 \\
\hline Winterslag SD & 1431 & 20.5 & -7.6 \\
\hline Winterslag T & 125 & 20.7 & -6.3 \\
\hline
\end{tabular}

Based on Table 4, a ranking of the aggregation methods could be created. The ranking is identical for both the optimized and the simulated IBPSA model, but differs slightly for the detailed IDEAS model. For this detailed model, the naive method also performs significantly worse, but the performance of all inertial methods is different. This needs further investigation within future work.

Finally, all three methods could be enhanced by taking a weighted average between the different dwellings, e.g. depending on the floor area or the volume. This extension, however, is left for future work.

To put these results into perspective, the differences between the two evaluation methods and the two building models are studied. Figure 6 illustrates the average $E_{S H}$ for the different evaluation methods and building models. Table 6 presents the differences between them on archetype, neighbourhood and district level, for the full simulation combined with the stochastic occupant model. The archetype simulations/optimizations are generally close to their respective full simulation/optimization. However, larger deviations are noted between the different evaluation methods. The optimizations with strict comfort requirements result in a pre-heating of the building and thus a higher $E_{S H}$ than the simulations that show some discomfort. The optimized $E_{S H}$ is on average $16.21 \%$ higher than the simulated $E_{S H}$ for the reduced-order IBPSA models. However, the dwellings of both neighbourhoods are not influenced to the same extent. The deviation of the optimized $E_{S H}$ compared to the simulated reduced-order IBPSA model for the newer dwellings (of Winterslag) is generally higher compared to the older dwellings (of Zwartberg). Additionally, the detailed IDEAS models generally result in a lower $E_{S H}$ than the reduced-order IBPSA models. The simulated $E_{S H}$ of the detailed IDEAS models is on average $10.13 \%$ lower compared to the reduced-order IBPSA models. Again, the dwellings of both neighbourhoods are not influenced to the same extent. Here, the deviation of the simulated $E_{S H}$ of the detailed IDEAS models compared to the reduced-order IBPSA models for the newer dwellings (of Winterslag) is generally lower compared to the older dwellings (of Zwartberg).

\subsubsection{Load duration curves}

When designing and optimizing district energy systems, not only $E_{S H}$ but also the temporal behaviour of $E_{S H}$ is important.
The temporal behaviour is assessed based on the LDCs of the different aggregation methods and is shown in Figure 9. In addition, the LDC of the full optimization using modesto is shown as a reference. The archetype optimizations based on the inertial aggregation method and the simplified inertial aggregation method match the full optimization most closely, whereas those based on the naive aggregation method and the inertial aggregation method with overheating deviate most. In general, the peak power range is approximated best, while larger deviations in the lower power range occur due to the optimization using a single building instead of many buildings. Typical for single building simulations/optimizations is the rounded shape with a rather sudden drop to zero at the lower end. When simulating/optimizing many buildings, these rounded shapes are aggregated into a much smoother decline in power.

The simulated reduced-order IBPSA models and detailed IDEAS models show a similar behaviour and are therefore not shown in this work.

\subsubsection{Computation times}

The computation times indicate the complexity of the three aggregation methods and should therefore also be considered when choosing a particular aggregation method. The simplified inertial method succeeds in calculating all building minimum temperature profiles in $40 \mathrm{~min}$, whereas the inertial method takes about 12 hours on the same computer. The naive method only takes around a minute. These calculations were performed on a Dell Precision 7920 Tower with two Intel Xeon Silver 4116 processors $(2.1 \mathrm{GHz}-3.0 \mathrm{GHz}$ Turbo, 12 cores) and $64 \mathrm{~GB}$ $2666 \mathrm{MHz}$ DDR4 RAM.

Although the simplified inertial aggregation method causes a certain loss in accuracy, it is preferred thanks to its simpler implementation and faster computation compared to the inertial aggregation method.

\subsubsection{Aggregation with a limited number of occupant behaviour profiles}

This section investigates the errors that occur when only a limited amount of data concerning the district is available. As explained in Section 2.3, an archetype setpoint temperature profile is now calculated using only a limited selection of set-point 

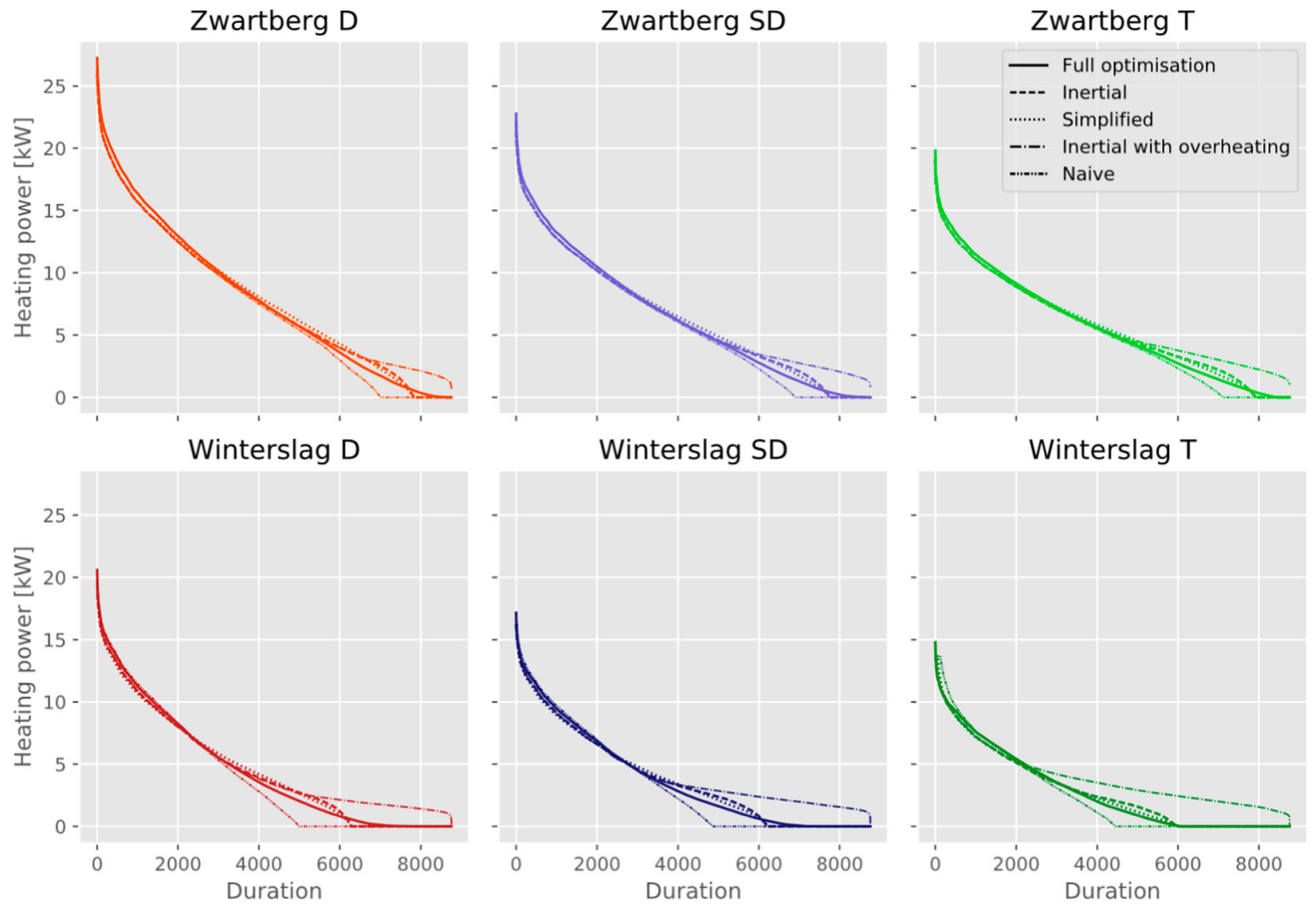

Figure 9. Comparison of LDCs obtained with different aggregation methods with the full optimisation case in modesto as a reference. The line styles for each of the six graphs represent the same methods and are explained in the figure legend.

temperature profiles. This selection is random and chooses 1, 5, $10,25,50,100$ or 200 set-point temperature profiles from the Winterslag $D$ district. Furthermore, such a selection is repeated 10 times for each number of set-point temperature profiles.

Figure 10 shows the relative error of $E_{S H}$ when comparing these random selections to the full optimization/simulation. If all 291 stochastically generated set-point temperature profiles are used, the error compared to the full simulation/optimization is limited to about $1 \%$, which is indicated by the black dotted line. As the number of included set-point temperature profiles becomes smaller, the variation in error becomes larger. Only using 1 set-point temperature profile can lead to an error of about $17.5 \%$, using 5 set-point temperature profiles can already reduce the error to about $7 \%$. Using 100 set-point temperature profiles reduces the error to about $1.5 \%$. It can be seen from the figure that the error decreases asymptotically to $1 \%$, when all set-point temperature profiles are included.

Figure 11 shows the LDCs of the full optimization, the simplified inertial method based on all 291 set-point temperature profiles and 10 variations of the simplified inertial method based on only 1 set-point temperature profile (left) and 5 set-point temperature profiles (right). This shows the spread and possible error in LDC caused by the random selection of set-point temperature profiles. It shows that the LDC generated with only a single set-point temperature profile can have large deviations from the full optimization. However, including a limited number of set-point temperature profiles already reduces these deviations

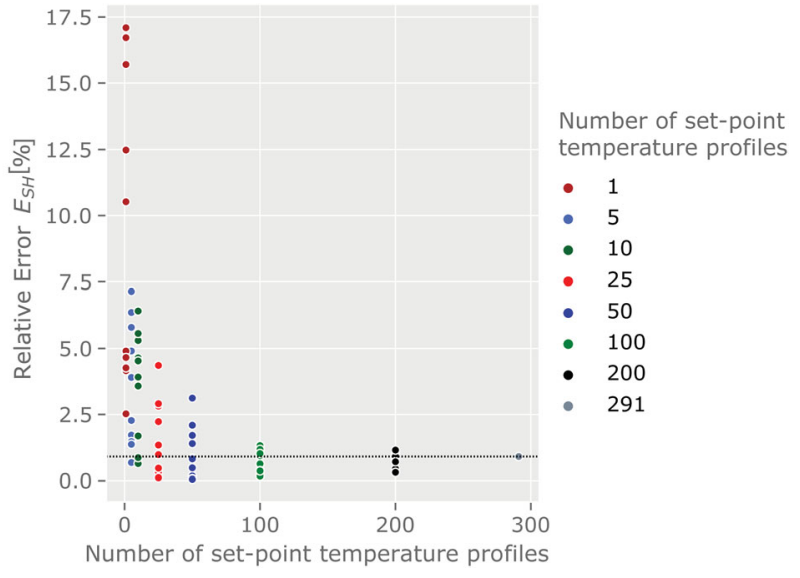

Figure 10. An overview of the relative error made on the Winterslag $D E_{S H}$ when using less set-point temperature profiles to generate the aggregated occupant behaviour profiles with the simplified inertial method. Each dot represents one optimisation. The colours of the dots indicate the number of buildings used to generate the aggregated occupant behaviour, although this is also indicated at the $x$-axis. The dotted line indicates the error made when using the simplified inertial method on all buildings within the cluster.

significantly. Nevertheless, the use of a limited number of occupant behaviour profiles within the simplified inertial aggregation method should be further investigated. The influence of including less occupant behaviour profiles highly depends on the variability between the different occupant behaviour profiles and the variability between the different dwellings that 

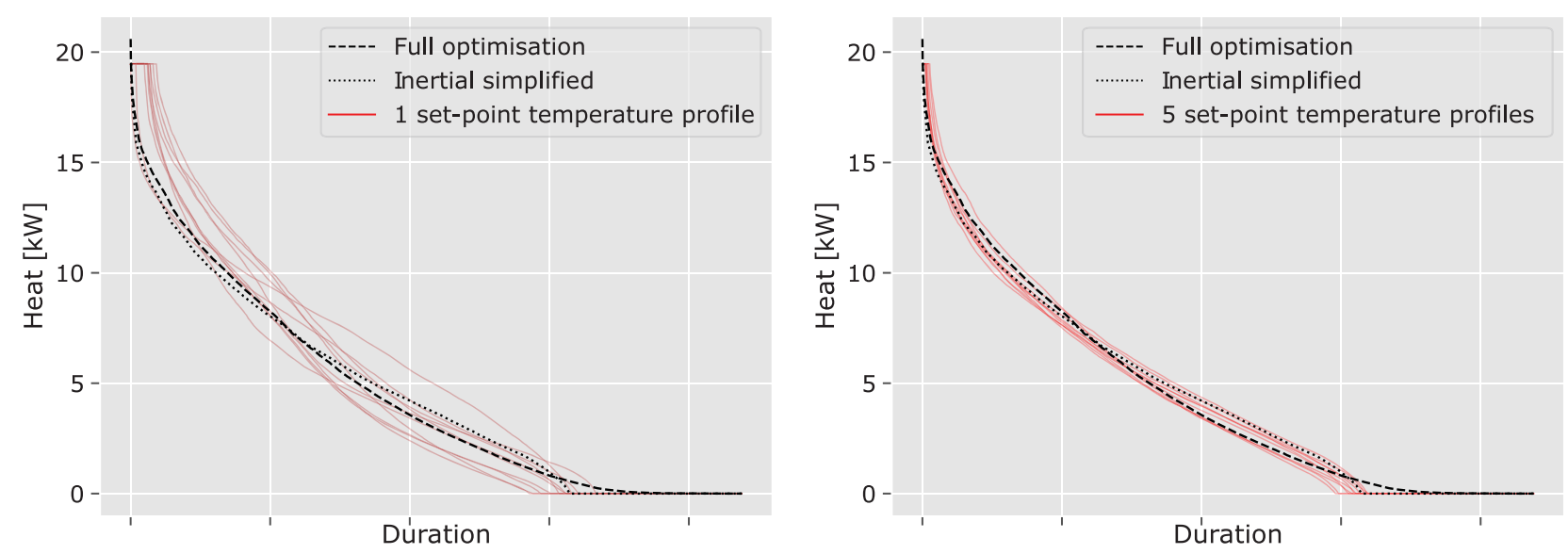

Figure 11. The Winterslag $D$ duration curves of the full optimisation case, the simplified inertial cases and the 10 cases using only 1 occupant behaviour profile (left) and 5 aggregated occupant behaviour profiles (right). This figure is made by using the optimisation method, yet the results of the simulations with both the detailed IDEAS models and the reduced-order IBPSA models show similar results.

follow a particular archetype. More insight in the variability of the occupant behaviour profiles generated by StROBe can be found in Baetens and Saelens (2016).

\section{Conclusions}

To avoid suboptimal decisions in the design and operation of district energy systems, simulations are often employed, as they aid in understanding the energy flow within these systems. However, the specific simulation approach depends on the scale of the study. For instance, to assess the location and capacity of central production and storage units, building-by-building simulations become computationally too expensive and archetype simulations provide a valuable alternative. In these archetype simulations, the building stock is categorized in a limited number of groups of buildings that are characterized by a similar energy performance and that can be represented by one archetype building. To define these archetype buildings, all required input data should be averaged, but this is not that intuitive for the occupant behaviour. Within the context of smart district energy systems, the variety in behaviour of the district is important, introducing the need to include stochastic occupant behaviour within the simulations. However, averaging stochastic occupant behaviour is not that straightforward, as the thermal inertia of the buildings causes them to react differently on their demanded set-point temperatures.

Within this context, this paper focuses on how stochastically generated occupant behaviour profiles can be aggregated for archetype simulations and on quantifying the resulting error by aggregating buildings and their stochastic occupant behaviour profiles into archetypes. Most aspects of the occupant behaviour can be simply averaged, but the set-point temperatures require a specific method, as the thermal inertia of the building should be included. Three different methods to aggregate stochastically generated set-point temperature profiles are compared in this work. The naive method just calculates the mean value of all inputs (i.e. internal heat gains and requested set-point temperature) across the selected profiles. The inertial method reflects that the set-point temperature cannot change immediately, such that the actual average temperature in all represented buildings will be higher than the result of the naive method during a decrease of the set-point. The simplified inertial method mimics the behaviour of the inertial method by doing sequential one-step simulations of the building model after the set-point decreases, until the cooling down reaches the new lower set-point. Hence, this method reduces the computation load significantly compared to the inertial method. An in-depth comparison is enabled by considering two evaluation methods (simulation and optimization), two building models (reducedorder IBPSA Modelica and detailed IDEAS Modelica) and two occupant models (deterministic and stochastic).

In all cases, the naive method underestimates $E_{S H}$ of the full simulation/optimization by roughly $2 \%$ for the deterministic occupant, but by approximately $7.5 \%$ for the stochastically generated occupant. Hence, especially for a stochastic occupant model, the naive method may not perform sufficiently. Including the thermal inertia of the dwellings improves the performance significantly. While considering the reducedorder IBPSA model, the inertial aggregation method deviates less than $1 \%$ and the simplified inertial aggregation method differs less than 3.7\% for $E_{S H}$, compared to the full simulation/optimization. Assessing the load duration curves for the different methods leads to a similar ranking. The inertial method corresponds best to the full simulation/optimization. Comparing computation times, the naive method is by far the fastest, taking only around a minute. The inertial method is slowest, while the simplified inertial method, needing about $40 \mathrm{~min}$ in this research, can speed up the inertial method by a factor of 18 . Considering the tradeoff between computational burden and accuracy, the simplified inertial aggregation method is preferred. Compared to the naive aggregation method, the simplified inertial aggregation method allows to gain approximately $4 \%$ in accuracy.

The performance of all aggregation methods can be put into perspective by comparing the use of different evaluation techniques as well as building models. Comparing the full simulations/optimizations, the optimized $E_{S H}$ is on average $16.21 \%$ higher than the simulated $E_{S H}$ for the reduced-order IBPSA models. The simulated $E_{S H}$ of the detailed IDEAS models is on average $10.13 \%$ lower compared to the reduced-order IBPSA models.

This comparison shows that the gain in accuracy is rather limited and may not always justify the extra computational power 
that is required to calculate the aggregated set-point temperature profile. Additionally, the inertial method was only applied to radiator heating systems, though slower heating devices, such as hydronic floors could make these methods even more complex. These slower systems will be the subject of future research.

Another limitation is the need for data describing the occupant behaviour. In this study, a toolbox to generate stochastic occupant behaviour was used to mimic real data. However, the collection of real data for these aggregation methods can be quite challenging. To estimate the consequences of missing data, this work also explored how many different stochastically generated occupant behaviour profiles should be included in the simplified inertial method, by randomly selecting 10 samples of $1,5,10,25,50,100,200$ or all set-point temperature profiles to obtain a representative aggregated set-point temperature profile for the archetype building Winterslag $D$. When including less set-point temperature profiles, the aggregation results deviate more from the full simulation/optimization, thus increasing the uncertainty on the estimation error. For the 10 samples of 5 set-point temperature profiles that were selected in this work for the archetype building Winterslag D, the additional error is limited to maximum $7.5 \%$. For the 10 samples of 100 set-point temperature profiles, the error is reduced to about $1 \%$. However, the influence of reducing the number of included occupant behaviour profiles highly depends on how many buildings are represented by the archetype, on the variability between the different occupant behaviour profiles and on the variability of the thermal performance between the different buildings. Therefore, further research in reducing the number of included occupant behaviour profiles is recommended.

By proposing the simplified inertial aggregation method, this work can help other researchers and energy simulation practitioners to improve their urban/district energy simulations when using archetype building models combined with stochastically generated occupant behaviour, while keeping the model complexity as low as possible.

\section{Notes}

1. In literature, archetype buildings are usually simulated with oversimplified occupant behaviour profiles (Happle, Fonseca, and Schlueter 2018). Oversimplified means that they either use a deterministic profile or one single occupant behaviour profile. This paper studies methods that aggregate multiple stochastically generated occupant behaviour profiles into one single representative profile.

2. A simulation is conceived as a step-by-step evaluation of a system model with fixed control rules or a PID controller. In contrast, a control optimisation controls the heat flows to the building over a longer period to minimize a preset objective function.

3. In a free floating scenario the space heating system is not turned on at all and the building temperatures depend only on the weather.

4. The archetype building model will still display some overheating, but using the inertial method with overheating would cause this model to inject heat even during this period, hence the overestimation.

\section{Acknowledgements}

The authors gratefully acknowledge the contribution of Vincent Reinbold with the visualization of the two neighbourhoods (Figure 5).

\section{Disclosure statement}

No potential conflict of interest was reported by the author(s).

\section{Nomenclature}

$E_{S H} \quad$ Annual energy demand for space heating $U$ Heat transfer coefficient $\left[\mathrm{W} /\left(\mathrm{Km}^{2}\right)\right]$

DES District Energy Systems

DHW Domestic hot water

HVAC Heating, ventilation and air conditioning

LDC Load Duration Curve

LOD Level of detail

R-C Resistance-capacitance

\section{Funding}

Ina De Jaeger holds a PhD grant fundamental research financed by the Research Foundation Flanders (FWO) and the Flemish Institute for Technological Research (VITO) (grant number: 11D0318N). The work of Annelies Vandermeulen and Bram van der Heijde is funded through a PhD Scholarship of the Flemish institure for Technological Research (VITO) (grant numbers: 1712,1505$)$.

\section{ORCID}

Ina De Jaeger (D) http://orcid.org/0000-0003-2200-2333

Annelies Vandermeulen (D) http://orcid.org/0000-0002-2696-2799

Bram van der Heijde (D) http://orcid.org/0000-0002-1389-1362

Lieve Helsen (D) http://orcid.org/0000-0002-9643-8204

Dirk Saelens (D) http://orcid.org/0000-0003-3450-5448

\section{References}

Allegrini, Jonas, Kristina Orehounig, Georgios Mavromatidis, Florian Ruesch, Viktor Dorer, and Ralph Evins. 2015. "A Review of Modelling Approaches and Tools for the Simulation of District-Scale Energy Systems." Renewable and Sustainable Energy Reviews 52: 1391-1404.

Baetens, Ruben, Roel De Coninck, Filip Jorissen, Damien Picard, Lieve Helsen, and Dirk Saelens. 2015. "OpenIDEAS an Open Framework for Integrated District Energy Simulations." In BS2015, 14th Conference of International Building Performance Simulation Association, 347-354.

Baetens, Ruben, and Dirk Saelens. 2016. "Modelling Uncertainty in District Energy Simulations by Stochastic Residential Occupant Behaviour." Journal of Building Performance Simulation 9 (4): 431-447.

Cuypers, Dieter, Birgit Vandevelde, Marlies Van Holm, and Stijn Verbeke. 2014. Belgische woningtypologie: nationale brochure over de TABULA woningtypologie. Technical Report.

Dassault Syst'emes AB. 2018. “Dymola 2019." Lund.

De Jaeger, Ina, Jesus Lago, and Dirk Saelens. 2018a. "A Probabilistic Approach to Allocate Building Parameters Within District Energy Simulations." In Urban Energy Simulation Conference 2018, Glasgow, UK.

De Jaeger, Ina, Glenn Reynders, Yixiao Ma, and Dirk Saelens. 2018b. "Impact of Building Geometry Description Within District Energy Simulations." Energy. 158.

De Jaeger, Ina, Glenn Reynders, and Dirk Saelens. 2017. "Impact of Spatial Accuracy on District Energy Simulations." Energy Procedia 132: 561-566.

Evins, Ralph, Kristina Orehounig, and Viktor Dorer. 2016. "Variability Between Domestic Buildings: The Impact on Energy Use." Journal of Building Performance Simulation 9 (2): 162-175.

Frayssinet, Löıc, Merlier Lucie, Kuznik Fŕed'eric, Hubert Jean-Luc, Maya Milliez, and JeanJacques Roux. 2017. "Modeling the Heating and Cooling Energy Demand of Urban Buildings at City Scale." Renewable and Sustainable Energy Reviews 81: 1-10.

Ghiassi, Neda, and Ardeshir Mahdavi. 2017. "Reductive Bottom-up Urban Energy Computing Supported by Multivariate Cluster Analysis." Energy and Buildings 144: 372-386.

Happle, Gabriel, Jimeno A Fonseca, and Arno Schlueter. 2018. "A Review on Occupant Behavior in Urban Building Energy Models." Energy and Buildings 174: 276-292.

Hong, Tianzhen, Da Yan, Simona D'Oca, and Chien fei Chen. 2017. "Ten Questions Concerning Occupant Behavior in Buildings: The Big Picture." Building and Environment 114: 518-530. 
Jorissen, F., G. Reynders, R. Baetens, D. Picard, D. Saelens, and L. Helsen. 2018. "Implementation and Verification of the IDEAS Building Energy Simulation Library." Journal of Building Performance Simulation 11 (6): 669-688.

Kensby, Johan, Anders Trüschel, and Jan-Olof Dalenbäck. 2015. "Potential of Residential Buildings as Thermal Energy Storage in District Heating Systems Results from a Pilot Test." Applied Energy 137: 773-781.

Kolbe, Thomas H, Gerhard Gröger, and Lutz Plümer. 2005. "CityGMLInteroperable Access to 3D City Models." Proceedings of the international symposium on Geo-Information for Disaster Management 883-900.

Lauster, M., J. Teichmann, M. Fuchs, R. Streblow, and D. Mueller. 2014. "Low Order Thermal Network Models for Dynamic Simulations of Buildings on City District Scale." Building and Environment 73: 223-231.

Lawrence, Thomas M, Marie-claude Boudreau, Lieve Helsen, Gregor Henze, Javad Mohammadpour, Doug Noonan, Dieter Patteeuw, Shanti Pless, and Richard T Watson. 2016. "Ten Questions Concerning Integrating Smart Buildings Into the Smart Grid." Building and Environment 108: 273-283.

Mavromatidis, Georgios, Kristina Orehounig, and Jan Carmeliet. 2018. "Uncertainty and Global Sensitivity Analysis for the Optimal Design of Distributed Energy Systems." Applied Energy 214: 219-238.

Modelica Association. 2017. "Modelica" -A Unified Object-Oriented Language for Systems Modeling Language Specification Version 3.4.".

OpenIDEAS. 2019. "An Open Framework for Integrated Building and District Energy Simulations." https://github.com/open-ideas.

Page, J., D. Robinson, N. Morel, and J. L. Scartezzini. 2008. "A Generalised Stochastic Model for the Simulation of Occupant Presence." Energy and Buildings 40 (2): 83-98.

Patteeuw, Dieter, and Lieve Helsen. 2014. "Residential Buildings with Heat Pumps, a Verified Bottom-Up Model for Demand Side Management Studies." In 9th International Conference on System Simulation in Buildings (SSB), Lìege, Lìege (Belgium), 1-19.

Protopapadaki, Christina, Glenn Reynders, and Dirk Saelens. 2014. "Bottomup Modelling of the Belgian Residential Building Stock: Impact of Building Stock Descriptions." In Proceedings of 9th International Conference on System Simulation in Buildings, Vol. 2, 1-21.
Reinhart, Christoph F., and Carlos Cerezo Davila. 2016. "Urban Building Energy Modeling: A Review of a Nascent Field." Building and Environment 97: 196-202.

Remmen, Peter, Moritz Lauster, Michael Mans, Marcus Fuchs, Tanja Osterhage, and Dirk Müller. 2018. "TEASER: An Open Tool for Urban Energy Modelling of Building Stocks." Journal of Building Performance Simulation 11 (1): 84-98.

Roulet, C.-A. 2002. "prEN-ISO 13790 - A Simplified Method to Assess the Annual Heating Energy Use in Buildings." ASHRAE Transactions: Symposia 108 (P): 911-918.

Swan, Lukas G., and V. Ismet Ugursal. 2009. "Modeling of End-Use Energy Consumption in the Residential Sector: A Review of Modeling Techniques." Renewable and Sustainable Energy Reviews 13 (8): 1819-1835.

Tahmasebi, Farhang, and Ardeshir Mahdavi. 2017. "The Sensitivity of Building Performance Simulation Results to the Choice of Occupants' Presence Models: a Case Study The Sensitivity of Building Performance Simulation Results to the Choice of Occupants' Presence Models: a Case Study." Journal of Building Performance Simulation 10 (5-6): 625-635.

Tardioli, Giovanni, Ruth Kerrigan, Mike Oates, James O'Donnell, and Donal P. Finn. 2018. "Identification of Representative Buildings and Building Groups in Urban Datasets Using a Novel Pre-processing, Classification, Clustering and Predictive Modelling Approach." Building and Environment 140: 90-106.

Tian, Wei, Yeonsook Heo, Pieter de Wilde, Zhanyong Li, Da Yan, Cheol Soo Park, Xiaohang Feng, and Godfried Augenbroe. 2018. "A Review of Uncertainty Analysis in Building Energy Assessment." Renewable and Sustainable Energy Reviews 93: 285-301.

Vandermeulen, Annelies, Bram van der Heijde, Dieter Patteeuw, Dirk Vanhoudt, Robbe Salenbien, and Lieve Helsen. 2018. "modesto - a Multi Objective District Energy Systems Toolbox for Optimization." In 5th International Solar District Heating Conference, Graz, apr.

Yao, Runming, and Koen Steemers. 2005. "A Method of Formulating Energy Load Profile for Domestic Buildings in the UK." Energy and Buildings 37 (6): 663-671. 